\title{
A Discrete Firefly Algorithm for the Scaffolding Modular Construction in Mega Projects
}

\author{
J.J. Liu ${ }^{a, b}$ L. Hou ${ }^{b}$ and X.Y. Wang ${ }^{b}$ \\ ${ }^{a}$ School of Science, China University of Petroleum, Beijing 102249, China \\ ${ }^{b}$ Australasia Joint Research Centre for BIM, Curtin University, Australia \\ E-mail: liujj@cup.edu.cn, lei.hou@curtin.edu.au,xiangyu.wang@curtin.edu.au
}

\begin{abstract}
-
Productivity is critical to large and complex capital projects in sectors like infrastructure and resources. It normally relates to a significant scheduling issue when pursuing both time and cost objectives. A poor planning of project schedule might incur both excessive labour cost and project delay. Looking at scaffolding activity, a foremost and prerequisite construction activity for the subsequence LNG equipment erection, this study identifies the present research gaps: very limited research emphasis has been placed on the impact of design of time-cost optimization in scaffolding, as well as the feasible solutions. Therefore, the practical guidance in scaffolding construction planning is treated as a critical research focal point in this study. Modular construction of scaffolding has been extensively implemented in LNG projects due to the risks of working on offshore platform and the enormous working area. The emphasis of this study therefore is placed on addressing time-cost tradeoff problems (TCTPs) of scaffolding modular construction. In particular, the questions of how to resolve the relatively multi-objective optimization issues using the Firefly algorithm and how to model the time and cost objective functions under specified constrains are answered. It is concluded that this paper, for the first time, looks into the complexity of scaffolding scheduling and gives solutions using such a robust optimization algorithm. In parallel, a simulation case study has been carrying out to test this algorithm based solution, to see how it can help scaffolding planners develop practical project schedules in either cost effective or time saving manners, or other trade-offs in between.
\end{abstract}

Keywords -

LNG; Time and cost trade-offs (TCTPs); Firefly Optimization Algorithm; Scaffolding

\section{Introduction}

Productivity is critical to increase Australias GDP. Analysis shows that lifting labour and capital productivity can result in AUD $\$ 90$ billion additional income per year by 2017 [1]. This is especially so for large and com- plex capital projects in sectors like infrastructure and resources. The data shows a $0.7 \%$ annual decline in productivity between 2005 and 2011 compared with a 2.4 $\%$ increase from 1993 to 1999 [1]. This underscores the urgency of getting productivity right and it is a priority area that can reap large rewards in future income growth [2]. Nowadays, scaffolding becomes an irremovable concern to industries across oil and gas, building, and infrastructure, considering the relatively low productivity and high labor shortage and cost. Poor design, planning and scheduling of scaffolding often lead to issues such as idling, rework, unnecessarily long travelling time between activities, which substantially reduce productivity. In mega projects that of great complexity such as offshore LNG (Liquefied Natural Gas) platform, high-rise building and infrastructure, scaffolding concerns a wide range of activities with different natures. Thus, construction process of scaffolding has a significant impact on the subsequent quality, safety and profitability of construction projects. However, the research works devoted to scaffolding issues may seldom or never be performed before and therefore are short of empirical data on guiding enacting offshore scaffolding schedule, despite its crucial importance [3].

In response to this, this paper focuses on scaffolding scheduling issues in an offshore LNG construction project, where the scaffold work is very large scale and involves huge amounts of factors such as materials, work crew, equipment, etc. The cost and efficiency from designing scaffolding schedule to its actual erection could be regarded as the focal point when conducting productivity research. Scaffolding normally applies modular construction in LNG projects. On the one hand, since laying LNG equipment takes up a lot of size and height, scaffolding therefore also has huge amount of work. The entire structure of scaffold, after modularization, can be divided into several small modules for parallel construction in different workshops, which saves time and workload. On the other hand, working on offshore platform is very risky as falling from the platform might incur drowning accident. Given the features of scaffolding modular con- 
struction, the objective of optimizing scaffolding schedule is to generate scaffolding design, erection and dismantling scheme, and their associated schedule and resource requirements and estimated cost by holistically consider multiple inputs (materials, work crew, equipment, etc.) under the constraints of design and progress of the building work, safety, workspace, budget, specification and codes, and so on. The emphasis should be placed on producing a series of outputs that have been optimised to inform decision-making.

\section{Literature Review}

Offshore LNG scaffolding engineering design problems generally involve several objectives. These objectives, related to the technique and economic performance of the engineering system, are potentially conflicting in nature. Multidisciplinary design optimization has grown to the point of gaining near universal recognition in its ability to lead to better designs [4]. Time and cost are two critical objectives of modular scaffolding construction, which are intricately concomitant to each other. To each workshop, using more workforces may speed up the progress of construction. But the total cost added up from each workshop would be definitely higher. Due to a limited research work on selecting options with corresponding time and cost to complete highly productive scaffolding activities, scaffolding scheduling is always accompanied by uncertainty. The emphasis is thus placed on working out the solution of allocating multiple appropriate resources for each modular construction to obtain the objective of overall time and overall cost. The timeCcost trade-off problems TCTPs are multi objective optimization problem [5]. The TCTPs address the project scheme options under the constraints of both project duration and project cost, so as to selects the best trade-offs to complete an activity. Given that randomness and fuzziness may co-exist in project scheduling problem, Ke and Liu [6] investigate various types of project scheduling problems using fuzzy activity, which can also be applied as the supplement of diverse algorithms to solve the software project scheduling problems[7]. To help project planners develop practical project schedules without impacting project quality, Kim et al. [8] take into account the potential quality loss cost in TCTPs, and propose a mixed integer linear programming model that considers the excessive crashing activities. In terms of the resourceconstrained (both renewable and non-renewable resources are as constraints) multi-mode scheduling solutions, Ant Colony Optimization (ACO) was studied by Li and Zhang [9], who manifest that, against other metaheuristic methods, this method is particularly beneficial for industry practitioners in real construction projects. Scheduling problems are normally concerned with assembly in flow- shop, such as queries scheduling and makespan, which is one of most difficult NP-complete problems. Allahverdi and Al-anzi [10] and Zhang et al. [11] compare Tabu Search (TS) approach with other types of algorithms such as particle swarm optimization (PSO) and EDD, and experimentally prove its quality (more effective) and performance (less error-prone). Simulated-Annealing (SA) can be applied in addressing the similar issue. According to Varadharajan and Rajendran [12], SA yields the most optimum and less computational solution in the net non-dominated multi-objective genetic local search than Elitist Non-dominated Genetic Algorithm (ENGA) [13] and (Gradual Priority Weighting) GPW approaches[14]. The population-based approaches incorporating differen$\mathrm{t}$ strategies for generating and improving a population of schedule have been very booming in achieving the similar objective, for example, the widely used Genetic Algorithm (GA) or Hybrid Genetic Algorithm (HGA) [15], [16], Ant Colony Optimization (ACO) [17], Particle Swarm Optimization Algorithm (PSOA)[18], Differential Evolution Algorithm (DEA) [19], Artificial Bee Colony Algorithm (ABCA)[20], etc. It is experimentally proved these population-based algorithms are averagely more competitive and efficient in searching local optimum in continuous and discrete multi-objective scheduling problems.

Firefly Algorithm (FA) is a novel algorithm which was firstly proposed in year 2010. Since then, numerous research works and literatures (detailed in [23]) have manifested its advantages over algorithms mentioned above in dealing with a wide range of issues. The study does not only establish the mathematical model of scaffolding modular construction in mega projects, but also propose a discrete self-adapted FA and demonstrate its viability in producing time-cost solutions.

\section{Problem Description and Mathematical Model}

Modular construction of scaffolding only considers each module is produced by an independent workshop, and the ultimate goal is the completion of each module so that the following final assembly can start. In the mathematical model, it assumes that each worker maintains the same productivity (working volume/time). Within each scaffolding module, there are a number of working step$\mathrm{s}$, where each working step requires a number of workers. Our objective function consists of two parts. First, there should incur the least cost after all the modules are completed. Second, there should be the minimum time consumption for the most time-consuming module. The assembly sequence within each module abides by precedence relationship (a group of task components are subject to order requirement). Resources are confined by 
the minimum and maximum number of workers available. Our mathematical model for the scaffolding modular construction problem is presented. First, notations used in our model are described and then the mathematical model is explained.

The notations are described here.

$I$ : maximum number of modules

$J_{i}$ : maximum number of assembly steps to produce scaffold module $i$

$P$ : set of precedence relations between procedures to product a scaffold module

$i$ : module index, $i=0,1, \cdots, I+1,(i=0$ or $I+1$ are dummy module)

$j$ : assembly step index in each module, $j=0,1, \cdots, J_{i}+1,\left(j=0\right.$ or $J_{i+1}$ are dummy steps)

$d_{i j}$ : duration of performing $j$-th assembly step in module $i$

$c_{i j}$ : cost of performing $j$-th assembly step in module $i$

$t_{i J}^{F}$ : finish times of last assembly $J$ in module $i$

$t_{i j}^{S}$ : start time of performing $j$-th assembly step in module $i$

$t_{i j}^{F}$ : finish time of performing $j$-th assembly

step in module $i$

$t_{i}^{D}$ : deadline of module $i$

$x_{i j}$ : resource variables, it can be continuous or discrete

$L$ : lower bound of resources

$U$ : upper bound of resources

$C_{\max }:$ maximum total cost

$T$ : time delay when finishing all the modules

The mathematical model of our problem (P) is presented as below:

$$
\begin{aligned}
& \min C_{\max }=\sum_{i=1}^{I} \sum_{j=1}^{J_{i}} c_{i j} \\
& \min T=\sum_{i=1}^{I} \max \left(0, t_{i, J}^{F}-t_{i}^{D}\right) \\
& t_{(i-1) J}^{S}+d_{(i-1) J} \leq t_{i 1}^{S}, \\
& t_{i(j-1)}^{S}+d_{i(j-1)} \leq t_{i j}^{S}, \\
& d_{i j}=f_{i j}^{d}\left(x_{i j}\right) \\
& c_{i j}=f_{i j}^{c}\left(x_{i j}\right) \\
& L \leq \sum_{i=1}^{I} \sum_{j=1}^{J_{i}} x_{i j} \leq U
\end{aligned}
$$

where $i=0,1, \cdots, I+1 ; j=0,1, \cdots, J_{i}+1$.

In above model, the objective function (1) shows the total modules cost. The second objective of our problem, as shown in objective function (2), is the total time to finishing all the modules, and should be minimized. The precedence relationships between activities and between workshops are described by (3) and (4), respectively. Equations (5) and (6) relate, respectively, the duration and the cost of performing $j$-th assembly step in module $i$ and to the resource variables. Formula (7) decides the scope of the resources. Based above mathematical model, a discrete Firefly algorithm will be applied to handle the scaffolding modular construction problem in next section.

\section{Firefly Algorithms (FA) for Addressing TCTPs}

In this section, a discrete FA algorithm will be present and then applied to TCTPs.

\subsection{Discrete Firefly Algorithm}

Firefly algorithm is a novel nature-inspired algorithm inspired by social behavior of fireflies. Fireflies are one of the most special, captivating and fascinating creature in the nature. By idealizing some of the flashing characteristics of fireflies, firefly-inspired algorithm was presented by Yang [21].

Firefly-inspired algorithms use the following three idealized rules:

(i) All fireflies are unisex which means that they are attracted to other fireflies regardless of their sex;

(ii) The degree of the attractiveness of a firefly is proportion to its brightness, thus for any two flashing fireflies, the less brighter one will move towards the brighter one and the more brightness means the less distance between two fireflies. If there is no brighter one than a particular firefly, it will move randomly;

(iii) The brightness of a firefly is determined by the value of the objective function. For a maximization problem, the brightness can be proportional to the value of the objective function.

In the discrete firefly algorithm, there are four important issues:

Attractiveness: In the firefly algorithm, the main form of attractiveness function $\beta(r)$ can be any monotonically decreasing functions such as the following generalized form:

$$
\beta(r)=\beta_{0} e^{-\gamma\left(r_{i j}\right)^{n}}, n \geq 1
$$

where $r$ is the distance between two fireflies, $\beta_{0}$ is the attractiveness at $r=0$ and $\gamma$ is a fixed light absorption coefficient.

Distance: The distance between any two fireflies $i$ and 
$j$ at $x_{i}$ and $x_{j}$ is the Cartesian distance as follows:

$$
r_{i j}=\left\|x_{i}-x_{j}\right\|=\sqrt{\sum_{k=1}^{d}\left(x_{i, k}-x_{j, k}\right)^{2}}
$$

where $x_{i, k}$ is the $k$-th component of the $i$-th firefly.

Movement: The movement of a firefly, $i$ is attracted to another more attractive (brighter) firefly $j$, is determined by

$$
\bar{x}_{i}=x_{i}+\beta(r) \times\left(x_{i}-x_{j}\right)+\alpha\left(r-\frac{1}{2}\right)
$$

where the second term is due to the attraction while the third term is randomization with being the randomization parameter and $\mathrm{r}$ is a random number generator uniformly distributed in $[0,1]$. In this paper, a outline update mode is applied in original FA, that means the light intensity is updated and the new position of firefly is evaluated after all the firefly finish moving.

The steps of the FA are given below [21]:

Step 1. Generate initial population of $n$ fireflies $x_{i},(i=1,2, \cdots, n)$ randomly each of which represents a candidate solution to the optimization problem with objective function of $f(x)$ and decision variables $x_{i}=x_{i 1}, x_{i 2}, \cdots, x_{i n}$.

Step 2. Compute light intensity using Eq. (8) for each firefly $\beta=\beta_{1}, \beta_{2}, \cdots, \beta_{n}$. The distance between fireflies is computed from Eq. (9).

Step 3. Move each firefly i toward other brighter fireflies using Eq. (10). If there is other brighter firefly move it randomly.

Step 4. Evaluate new solutions and update light intensity.

Step 5. Rank the fireflies and find the current best solution.

Step 6. Repeat steps 2-5 until termination criterion is satisfied.

Based on the effectiveness of the firefly algorithm in optimizing continues problems, it is predictable that this algorithm would be impressive to solve discrete optimization problems which creates the motivation for proposing a discrete firefly algorithm. Sayadi et al. [22] developed a discrete version of FA (DFA) which can efficiently solve NP-hard scheduling problems, while a detailed analysis has demonstrated the efficiency of FA over a wide range of test problems, including multi-objective load dispatch problems. Furthermore, DFA can also solve scheduling and travelling salesman problem in a promising way [23].

Discretization: When the firefly $i$ moves toward firefly $j$, the position of firefly $i$ is changed from a binary number to a real number. Therefore, we must replace this

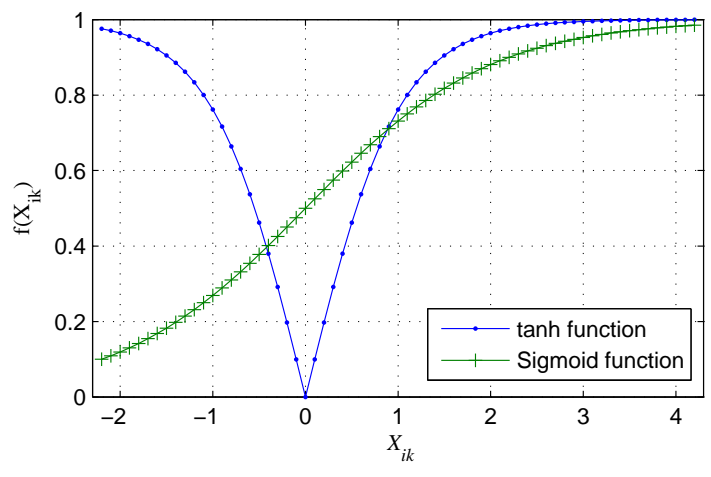

Figure 1. Variation of $X_{i k}$ and $f\left(X_{i k}\right)$

real number by a binary number. The following sigmoid function restricts $f\left(x_{i k}\right)$ to be in the interval $[0,1]$ :

$$
f\left(x_{i k}\right)=\frac{1}{1+e^{-x_{i k}}}
$$

or

$$
f\left(x_{i k}\right)=\tan \left|x_{i k}\right|=\frac{e^{2\left|x_{i k}\right|}-1}{e^{2\left|x_{i k}\right|}+1}
$$

where $f\left(x_{i k}\right)$ denotes the probability of $x_{i k}$ equalling 1 (See Figure 1). Then we can map interval $(0,1)$ into the integral interval which is the feasible area of the problem. The results of numerical experiments show that Eq.(12) is better than Eq.(11) in most cases. So the Eq.(12) is used in this paper.

The steps of the DFA can be summarized as the pseudo code shown in Algorithm 1.

\subsection{An adaptive Discrete FA for addressing TCTPs}

For a better performance of DFA, an adaptive search strategy is proposed which consists of the parameters varies with the current iteration number $t$.

$$
\alpha=\alpha_{0}-\frac{1}{1+e^{-\left(t-\frac{t_{\max }}{2}\right)}}
$$

where $t_{\max }$ is the number of the maximum generation, $\alpha_{0}$ is the maximum value of $\alpha$. Figure 2 demonstrates the variation of $\alpha$ with respect to $t$. This adaptive variation can play a balance adjustment of convergence and diversity.

In our TCTPs of the scaffolding modular construction problem there is one kinds of variable $x_{i j}$, which denotes the number of works performing $j$-th assembly step in modular $i$. Both minimized objective functions and four constraints are taken into account in the mathematical model. The linear weight method is used to convert two objective functions into single objective by the following equation:

$$
F\left(x_{i j}\right)=\omega_{1} C_{\max }+\omega_{2} T
$$




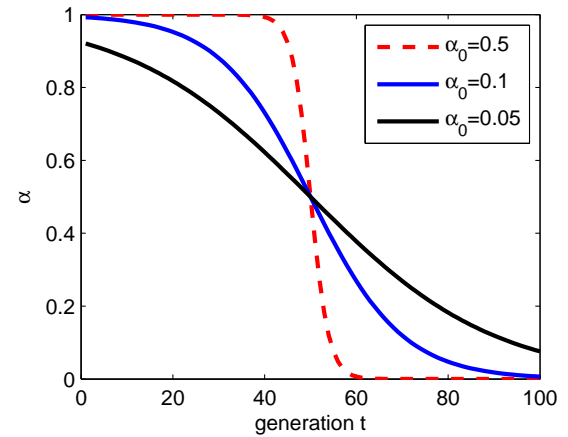

Figure 2. Variation of $\alpha$ with respect to different $t$

where $\omega_{1}$ and $\omega_{2}$ are weights decided by user according to his expect on each objective function. Generally, $\omega_{1}+\omega_{2}=1$. The position for the $k$ th firefly in the $t$-th generation can be denoted as $x_{k i j}^{t}$. For convenience, $x_{k i j}^{t}$ is expressed in a vector $\left(x_{k 11}^{t}, \cdots, x_{k 1 J}^{t}, \cdots, x_{k i 1}^{t}, \cdots, x_{k i J}^{t}, \cdots, x_{k I J}^{t}\right)$. As to dealing the constraints, we adapt the technique of the penalty function to convert the constrained optimization problem into unconstrained optimization.

$$
\min P\left(x_{i j}\right)=F\left(x_{i j}\right)+G\left(x_{i j}\right)
$$

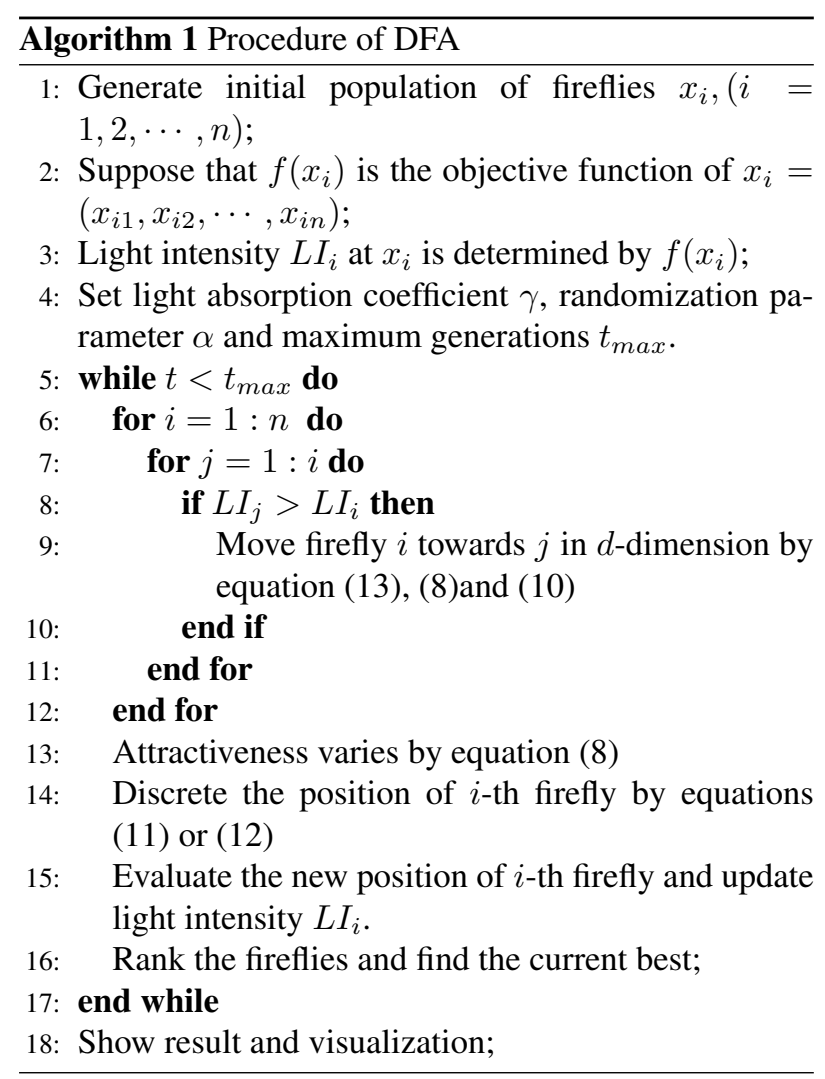

where

$$
\begin{aligned}
G\left(x_{i j}\right)= & \mu \sum_{i=1}^{I} \sum_{j=1}^{J} \max \left\{0, t_{i(j-1)}^{S}+d_{i(j-1)}-t_{i j}^{S}\right\} \\
& +\lambda \sum_{i=1}^{I} \max \left\{0, t_{(i-1) J}^{S}+d_{(i-1) J}-t_{i 1}^{S}\right\}
\end{aligned}
$$

and $\mu, \lambda \rightarrow \infty$.

Hence the discrete Firefly algorithm can perform for the scaffolding modular construction problem.

\subsection{Case Study}

A real 3-module LNG construction case study derived from an offshore LNG platform is selected to fit into the proposed model. Here, assembly steps had several options of time and resources. The module $i$, assembly step $j$, precedence relationships Pre., cost of each step $c_{i j}$, duration of each step $d_{i j}$, and deadline $t_{i}^{D}$ of each module

\begin{tabular}{|c|c|c|c|c|c|c|}
\hline$i$ & $j$ & Description & Pre. & $c_{i j}$ & $d_{i j}$ & $t_{i}^{D}$ \\
\hline \multirow{4}{*}{1} & 1 & cantilever & - & $3 x_{11}+2$ & $24-5 x_{11}$ & \\
\hline & 2 & vertical & 01 & $3 x_{12}+1$ & $20-3 x_{12}$ & 15 \\
\hline & & reinforcing & & & & \\
\hline & 3 & post & 02 & $2 x_{13}+3$ & $15-x_{13}$ & \\
\hline \multirow{5}{*}{2} & 1 & horizontal & 13 & $3 x_{21}+1$ & $15-2 x_{21}$ & \\
\hline & & reinforcing & & & & 12 \\
\hline & & & & & & \\
\hline & 2 & $\begin{array}{l}\text { traversing } \\
\text { lever }\end{array}$ & 13 & $x_{22}+2$ & $10-x_{22}$ & \\
\hline & 3 & Joist & $21-22$ & $2 x_{23}$ & $15-3 x_{23}$ & \\
\hline \multirow{5}{*}{3} & 1 & cross brace & 23 & $2 x_{31}+1$ & $12-x_{31}$ & \\
\hline & 2 & planking & $01-31$ & $2 x_{32}+5$ & $18-2 x_{32}$ & 10 \\
\hline & 3 & guard sys- & $01-31$ & $x_{33}+4$ & $14-x_{33}$ & 10 \\
\hline & & tem & & & & \\
\hline & 4 & safety net & 01-31 & $x_{34}+3$ & $15-x_{34}$ & \\
\hline
\end{tabular}
are detailed in Table 1.

Table 1. Case information.

Utilizing data or expresses into optimization model (15), we applied the presented DFA to find the solution of problem $(\mathrm{P})$. Where the parameters are preset as follow: population size is 40, Max Generation is $100, L=0$ and $U=50$. Discretization is performed by using of equation (12), and then the real variables $x_{i j}$ are mapped into integral interval $[1, U]$. After a run of the proposed algorithm, a quasi-optimal duration, cost, and workers allocation of the problem is presented in Table 2.

Figure 3 shows the performance of the algorithm for the case and Figure 4 shows the pareto front of the case. 
Table 2. Optimal assembly duration and cost.

\begin{tabular}{|c|c|c|c|c|c|}
\hline$i$ & $j$ & Description & Duration(day) & $\operatorname{Cost}\left(10^{5}\right)$ & Allocatic \\
\hline \multirow{4}{*}{1} & 1 & cantilever & 19 & 5 & 1 \\
\hline & 2 & vertical & 18 & 4 & 1 \\
\hline & & $\begin{array}{l}\text { reinforcing } \\
\text { tube }\end{array}$ & & & \\
\hline & 3 & post & 13 & 1 & 1 \\
\hline \multirow{4}{*}{2} & 1 & horizontal & 5 & 6 & 5 \\
\hline & & reinforcing & & & \\
\hline & 2 & traversing & 7 & 5 & 3 \\
\hline & 3 & Joist & 1 & 5 & 5 \\
\hline \multirow{5}{*}{3} & 1 & cross brace & 11 & 3 & 1 \\
\hline & 2 & planking & 9 & 10 & 5 \\
\hline & 3 & guard sys- & 6 & 12 & 4 \\
\hline & & tem & & & \\
\hline & 4 & safety net & 6 & 2 & 1 \\
\hline \multicolumn{4}{|c|}{ Sum } & 53 & 27 \\
\hline
\end{tabular}

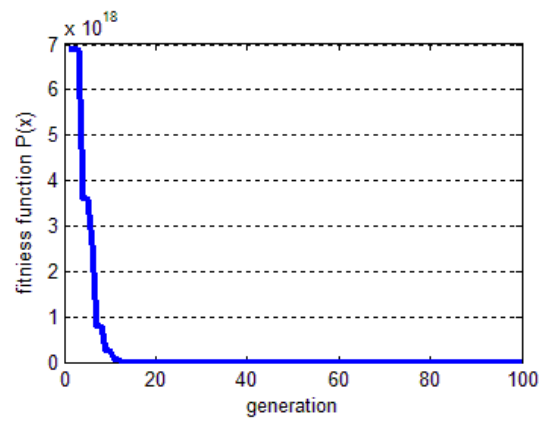

Figure 3. the convergence curve of results

\section{Conclusions}

The paper studied the project scheduling problem with modular construction activity. For obtaining the minimal total modules cost and minimal total time to finishing al1 the modules, a multi-objective constrained optimization model was established and a modified intelligent algorith$\mathrm{m}$ (DFA) to find the optimal solution of this model was proposed. FA is a excellent intelligent algorithm which has been successfully applied in several real discrete optimisation problems [23]. The focal point of the paper is to investigate the scaffolding modular construction issues, which belong to a series of discrete TCTPs. Through a substantial project-based case study, the results manifest the credibility of the optimisation by producing a better solution of workforce allocation for the sake of time and cost balance.

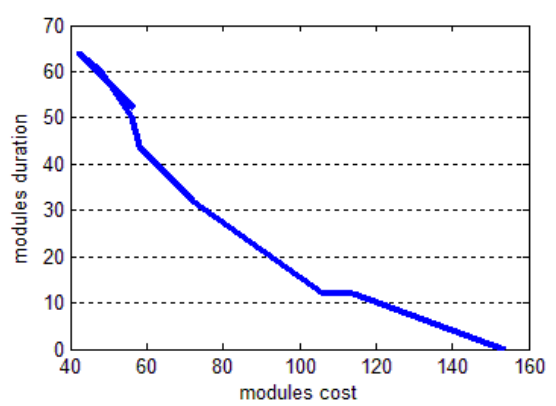

Figure 4. Pareto front obtained by DSA

\section{Acknowledgements}

Part of this research was supported under Australian Research Council Linkage Project scheme ( project number: LP130100451).

\section{References}

[1] Taylor, C., Bradley, C., Dobbs, R., Thompson, F. and Clifton, D. Beyond the boom: Australia's productivity imperative. McKinsey Global Institute, McKinsey and Company, Sydney, 2012.

[2] Shann, E. W. Maximising growth in a mining boom: Minerals Council of Australia, 2012.

[3] Rubio-Romero, J. C., Carmen Rubio Gmez, M. and Carrillo-Castrillo, J. A. Analysis of the safety conditions of scaffolding on construction sites. Safety Science, 55, 160-164, 2013.

[4] Keane, A. and Nair, P. Computational approaches for aerospace design: the pursuit of excellence: John Wiley \& Sons, 2005.

[5] Feng, C.-W., Liu, L. and Burns, S. A. Using genetic algorithms to solve construction time-cost trade-off problems. Journal of Computing in Civil Engineering, 11(3), 184-189, 1997.

[6] Ke, H. and Liu, B. Fuzzy project scheduling problem and its hybrid intelligent algorithm. Applied Mathematical Modelling, 34(2), 301-308, 2010.

[7] Huang, W., Ding, L., Wen, B. and Cao, B. Project scheduling problem for software development with random fuzzy activity duration times Advances in Neural NetworksCISNN 2009. (pp. 6069): Springer, 2009.

[8] Kim, J., Kang, C. and Hwang, I. A practical approach to project scheduling: considering the potential quality loss cost in the timeCcost tradeoff prob- 
lem. International Journal of Project Management, 30(2), 264-272, 2012.

[9] Li, H. and Zhang, H. Ant colony optimization-based multi-mode scheduling under renewable and nonrenewable resource constraints. Automation in Construction, 2013.

[10] ALLAHVERDI, A. and AL-ANZI, F. S. A PSO and a Tabu search heuristics for the assembly scheduling problem of the two-stage distributed database application. Computers \& Operations Research, 33, 1056-1080, 2006.

[11] Zhang, C., Li, P., Guan, Z. and Rao, Y. A tabu search algorithm with a new neighborhood structure for the job shop scheduling problem. Computers \& Operations Research, 34(11), 3229-3242, 2007.

[12] Varadharajan, T. and Rajendran, C. A multiobjective simulated-annealing algorithm for scheduling in flowshops to minimize the makespan and total flowtime of jobs. European Journal of Operational Research, 167(3), 772-795, 2005.

[13] Baghi, T. P. Multiobjective scheduling by genetic algorithms: Springer, 1999.

[14] Chang, P.-C., Hsieh, J.-C. and Lin, S.-G. The development of gradual-priority weighting approach for the multi-objective flowshop scheduling problem. International Journal of Production Economics, 79(3), 171-183, 2002.

[15] Pezzella, F., Morganti, G. and Ciaschetti, G. A genetic algorithm for the flexible job-shop scheduling problem. Computers \& Operations Research, 35(10), 3202-3212, 2008.

[16] Gonalves, J. F., de Magalhes Mendes, J. J. and Resende, M. c. G. A hybrid genetic algorithm for the job shop scheduling problem. European Journal of Operational Research, 167(1), 77-95, 2005.

[17] Merkle, D., Middendorf, M. and Schmeck, H. Ant colony optimization for resource-constrained project scheduling. Evolutionary Computation, IEEE Transactions on, 6(4), 333-346, 2002.

[18] Jarboui, B., Damak, N., Siarry, P. and Rebai, A. A combinatorial particle swarm optimization for solving multi-mode resource-constrained project scheduling problems. Applied Mathematics and Computation, 195(1), 299-308, 2008.

[19] Pan, Q.-K., Tasgetiren, M. F. and Liang, Y.-C. A discrete differential evolution algorithm for the permutation flowshop scheduling problem. Computers \& Industrial Engineering, 55(4), 795-816, 2008.
[20] Karaboga, D. and Basturk, B. A powerful and efficient algorithm for numerical function optimization: artificial bee colony (ABC) algorithm. Journal of global optimization, 39(3), 459-471, 2007.

[21] Yang, X.-S. Nature-inspired metaheuristic algorithms: Luniver Press, 2010.

[22] Sayadi, M., Ramezanian, R. and Ghaffari-Nasab, N. A discrete firefly meta-heuristic with local search for makespan minimization in permutation flow shop scheduling problems. International Journal of Industrial Engineering Computations, 1(1), 2010.

[23] Yang, X. S. and He, X. Firefly algorithm: recent advances and applications. International Journal of Swarm Intelligence, 1(1), 36-50, 2013. 